\title{
ON GENERALIZING BOY'S SURFACE: CONSTRUCTING A GENERATOR OF THE THIRD STABLE STEM
}

\author{
J. SCOTT CARTER
}

\begin{abstract}
An analysis of Boy's immersion of the projective plane in 3-space is given via a collection of planar figures. An analogous construction yields an immersion of the 3-sphere in 4-space which represents a generator of the third stable stem. This immersion has one quadruple point and a closed curve of triple points whose normal matrix is a 3-cycle. Thus the corresponding multiple point invariants do not vanish. The construction is given by way of a family of three dimensional cross sections.
\end{abstract}

Stable homotopy groups can be interpreted as bordism groups of immersions. The isomorphism between such groups is given by the Pontryagin-Thom construction on the normal bundle of representative immersions. The self-intersection sets of immersed $n$-manifolds in $\mathbf{R}^{n+1}$ provide stable homotopy invariants. For example, Boy's immersion of the projective plane in 3-space represents a generator of $\Pi_{3}^{s}\left(P^{\infty}\right)$; this can be seen by examining the double point set. In this paper a direct analog of Boy's surface is constructed. This is an immersion of $S^{3}$ in 4-space which represents a generator of $\Pi_{3}^{s}$; the self-intersection data of this immersion are as simple as possible. It may be that appropriate generalizations of this construction will exemplify a nontrivial Kervaire invariant in dimension 30,62 , and so forth.

I. Background. Let $i: M^{n} \leftrightarrow \mathbf{R}^{n+1}$ denote a general position immersion of a closed $n$-manifold into real $(n+1)$-space. Assume that $i$ and $M$ are smooth, and that $\mathbf{R}^{n+1}$ is given the standard differential structure. By applying the Pontryagin-Thom construction on the normal bundle of $(i, M)$, one obtains a stable homotopy class in $\Pi_{n+1}^{s}\left(P^{\infty}\right)$. Here $P^{\infty}$ denotes the infinite real projective space, an Eilenberg-Mac Lane space of type $K(\mathbf{Z} / 2,1) .\left(\Pi_{j}^{s}(X)=\lim _{k}\left[S^{k+j}, S^{k} X\right]\right.$, for $X$ a locally compact Hausdorff space; $S^{k}(\cdot)$ is the reduced suspension.) If the pair $(i, M)$ is varied by a bordism, the same homotopy class arises. Furthermore, if $M$ is oriented, then the corresponding homotopy class lies in $\Pi_{n}^{s}\left(=\Pi_{n+1}^{s}\left(S^{1}\right)\right.$ by adjointness). Finally any class in $\Pi_{n+1}^{s}\left(P^{\infty}\right)$ or $\Pi_{n}^{s}$ may be realized in this way. These isomorphisms were noticed first by Wells [20], see also [16] for a modern proof.

Stable homotopy has recently been approached from the immersion point of view (see [5, 7, 14, and 19] for example). Since immersions are concrete geometric objects, this new approach brings into focus many of the terse ideas of homotopy theory. One can distinguish immersions by means of their self-intersection data; e.g. the

Received by the editors March 9, 1984.

1980 Mathematics Subject Classification (1985 Revision). Primary 57R42, 57R65, 55Q45, 57N35.

C1986 American Mathematical Society $0002-9947 / 86 \$ 1.00+\$ .25$ per page 
figure 8 immersion of the circle into the plane differs from an embedding in a fundamental way. In fact the self-intersection set of immersions do engender stable homotopy invariants which are classically understood.

The self-intersection invariants

$$
\begin{array}{r}
\varphi_{j}: \Pi_{n+1}^{s}\left(P^{\infty}\right) \rightarrow \Pi_{n+1}^{s}\left(D_{j} P^{\infty}\right) \text { and } \psi_{j}: \Pi_{n+1}^{s}\left(S^{1}\right) \rightarrow \Pi_{n+1}^{s}\left(D_{j}\left(S^{1}\right)\right), \\
j=1, \ldots, n+1,
\end{array}
$$

are defined as follows. Pick an immersion $\left(i: M^{n} \rightarrow \mathbf{R}^{n+1}\right)$ representing a homotopy class, and assign to the bordism class of $(i, M)$, the bordism class of the $j$-fold self-intersection data. These data consist of (1) an $(n+1-j)$-manifold, $M(j)$ (the $j$-tuple manifold), (2) a continuous map $g: M(j) \rightarrow B G$ into a classifying space ( $G$ is the group of (signed, if $M$ is not oriented) permutation matrices on $j$ letters), and (3) a vector bundle isomorphism $T M(j) \oplus g^{*}(\theta) \stackrel{\triangleq}{\rightarrow} \varepsilon^{n+1}$ (where $\theta$ is the vector bundle over $B G$ induced by the given representation, and $\varepsilon^{n+1}$ is the trivial $(n+1)$-plane bundle over $M(j))$. The map $g$ and the bundle isomorphism arise from considering the image of the normal bundle of $i$ restricted to the image of $M(j)$ in $\mathbf{R}^{n+1}$. The bordism groups of such triples are Pontryagin-Thom isomorphic to the stable homotopy groups indicated, as was shown in [5 and 16]. The homomorphisms $\varphi_{n+1}$ and $\psi_{n+1}$ count the number of $(n+1)$-tuple points modulo 2. With regard to these invariants we have the following theorems.

THEOREM (3.5 OF [7]). If $p$ is a prime, $0 \leqslant p \leqslant n+1$, the p-fold self-intersection invariant

$$
\psi_{p}: \Pi_{n+1}^{s}\left(S^{1}\right) \rightarrow \Pi_{n+1}^{s}\left(D_{j} S^{1}\right)
$$

is injective on the pth component.

THEOREM. There exists an immersion $i: M^{n} \rightarrow \mathbf{R}^{n+1}$ with one $(n+1)$-tuple point only if $n=0,1,2,3,5,6$ or $2^{j}-3$. In case $n=4 k+1$ there is such an immersion if there is a framed $(n+1)$-manifold of Kervaire invariant 1 . Such an immersion is known to exist when $n=0,1,2,3,5,6,13,29$, or 61 .

This second theorem is due to several people: Eccles [7, 8], Freedman [9], Lannes [17], and Banchoff [1]. P. J. Eccles showed the $(n+1)$-tuple invariant vanished on oriented immersions for $n>3$, and demonstrated the agreement of the $(n+1)$ invariant with the Kervaire invariant when $n=4 k+1$ and immersions are unoriented. Lannes showed that $n=3$ is the only dimension congruent to 3 modulo 4 for which such an immersion exists. The proofs of these theorems rely heavily on the machinery of stable homotopy; in particular, explicit immersions are seldom constructed. In fact only in the cases $n=1,2,3$, and 5 are explicit immersions known.

Boy's surface generates $\Pi_{3}^{s}\left(P^{\infty}\right) \simeq \mathbf{Z} / 8$, and this can easily be seen by evaluating the double point invariant on the surface. The third stable stem $\Pi_{3}^{s}$ is isomorphic to $\mathbf{Z} / 24$. An element of order eight is obtained by Koschorke's figure 8 construction on Boy's surface [14]. Capping off 2-sphere eversions at both ends with 3-balls yields a homomorphism from the group of regular homotopy classes of eversions to $\Pi_{3}^{s}$. Hughes [11] has shown that this homomorphism is surjective, and the Max-Banchoff 
[18] sphere eversion represents a generator. However, this method of constructing immersed closed manifolds does not generalize.

In this paper a generator of $\Pi_{3}^{s}$ is explicitly constructed as an immersed handlebody. The self-intersection sets consist of a single quadruple point, a closed triple point curve with a one-third twist in the normal frame, and a double-point manifold which is an immersed $\mathbf{R} \mathbf{P}^{2}$. The intrinsic manifold is $S^{3}$. According to the first theorem quoted above (3.4 of [7]), the self-intersections must be at least as complicated as this. It seems reasonable that the methods given here generalize to obtain immersions with one $(n+1)$-tuple point whenever possible. Moreover, the vanishing of the $(n+1)$-tuple invariant might be detected by attempting this construction.

The author wishes to thank his colleagues at the University of Texas for many useful conversations. Thanks are also due to Professor W. S. Massey who originally suggested this procedure. Finally, thanks go out to the referee for his helpful comments.

II. Boy's surface. Boy's surface [2] is an immersion of $\mathbf{R} \mathbf{P}^{2}$ in $\mathbf{R}^{3}$ with one triple point. A theorem of Banchoff shows the number of triple points of an immersed surface in 3-space is congruent modulo 2 to the Euler characteristic of the surface [1]. Below, Boy's immersion is described as the image of a nonstandard handlebody decomposition of the projective plane. In this decomposition there are three 0-handles, six 1-handles, and four 2-handles. The image of any of the 0-handles is a coordinate 2-disk. Thus the images of the 0-handles intersect at the origin, and any two intersect in a coordinate arc. Appropriate pairs of 1-handles intersect along arcs which connect the various endpoints of the coordinate arcs. Finally, all 2-handles are attached so as not to lie in the self-intersection sets. The key to understanding the immersion constructed in §III is to understand Boy's immersion.

Consider in $\mathbf{R}^{3}$ the square of radius $r$ (in the sup norm)

$$
\Gamma_{j}(r)=\left\{\left(x_{1}, x_{2}, x_{3}\right): x_{j}=0,\left|x_{i}\right| \leqslant r\right\}, \quad j=1,2,3
$$

for brevity, $\Gamma_{j}(1)=\Gamma_{j}$. The squares $\Gamma_{1}, \Gamma_{2}, \Gamma_{3}$ intersect pairwise along coordinate arcs of length 2 , and $\Gamma_{1} \cap \Gamma_{2} \cap \Gamma_{3}=(0,0,0)$; see Figure 1 .

Thus we have an immersion in $\mathbf{R}^{3}$ of three squares; these are 0 -handles of $\mathbf{R} \mathbf{P}^{2}$. Let $e_{j}$ denote the $j$ th unit vector. An immersion of a closed manifold is desired, so "to close" this, the self-intersection sets are closed sequentially. (To close an immersion with boundary, we surger the boundary by attaching ambient handles until the boundary is spherical, and then remove each sphere component by adding a ball.)

The boundary of the double point set consists of the six points $( \pm 1,0,0)$, $(0, \pm 1,0)$, and $(0,0, \pm 1)$; therefore this boundary is spherical. In the square $\Gamma_{j+2}(5)$ join the point whose $j$ th coordinate is +1 to the point whose $(1+j)$ th coordinate is -1 by an arc $p_{j}$ as shown in Figure 2 . Throughout this section subscripts are reduced modulo 3. The normal space, $\nu\left(p_{j}(t)\right)$, of $p_{j}(t)$ is spanned by the vectors $e_{j+2}$ and $p_{j}^{\perp}(t)$ where $p_{j}^{\perp}(t)$ is the unit normal of $p_{j}$ in $\Gamma_{j+2}$. Each $p_{j}$ is the intersection of two 1-handles parametrized as follows. 


$$
\begin{gathered}
h_{j, 0} ; h_{j, 1}:[0,1] \times[-1,1] \rightarrow \mathbf{R}^{3}, \\
h_{j, 0}(s, t)=p_{j}(s)+t p_{j}{ }^{\perp}(s), \\
h_{j, 1}(s, t)=p_{j}(s)+t e_{j+2} .
\end{gathered}
$$

The handle $h_{j, 0}$ connects the eastern edge of $\Gamma_{j+2}$ to the southern edge of $\Gamma_{j+2}$. The handle $h_{j, 1}$ connects the northern edge of $\Gamma_{j+1}$ to the western edge of $\Gamma_{j}$. This is illustrated in Figure 3. Notice the orientation of $\Gamma_{2}$ is given by $\left[e_{3}, e_{1}\right]$.

For each $j=1,2,3$, and for an appropriate choice of curves $p_{j},\left\{h_{j, 0}(s,-1)\right\}$ forms a simple closed curve contained in $\Gamma_{j+2}(5)$; each such curve bounds a disk (shaded in Figure 3) whose boundary is a spherical component of the boundary of the immersion given so far. These three "triangular" disks are then attached to yield an immersed Möbius band as in Figure 4. (This is a facsimile of a drawing in Eccles's paper [6].)
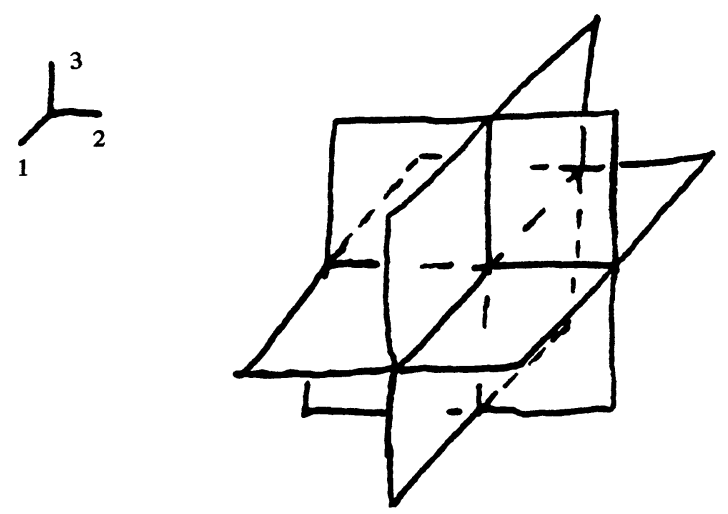

FIGURE 1

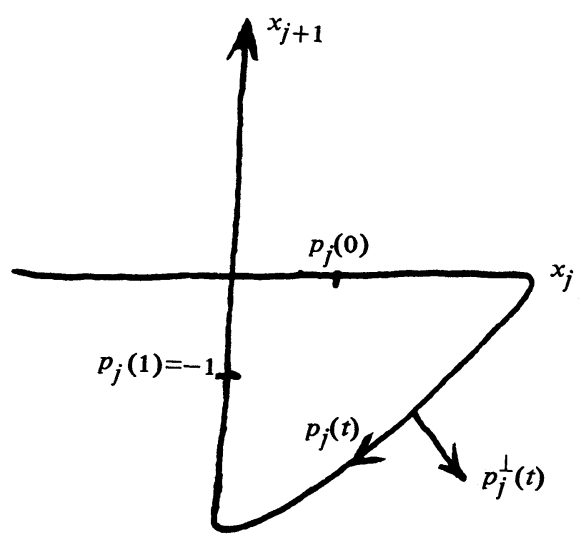

Figure 2 
In order to complete the construction of Boy's immersion the remaining boundary component must be closed. Closing this boundary is easy if one can see in three dimensions (see Figure 8). We prefer to consider the problem with two-dimensional eyes, for in §III we are not equipped with four-dimensional eyes.

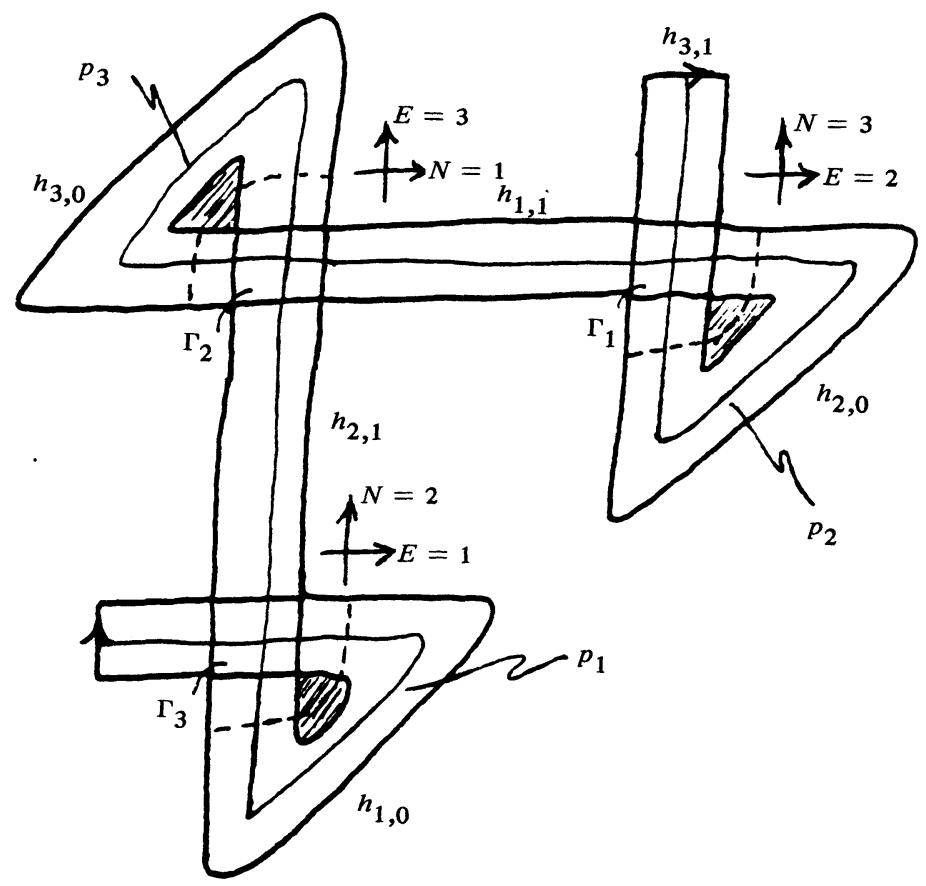

Figure 3

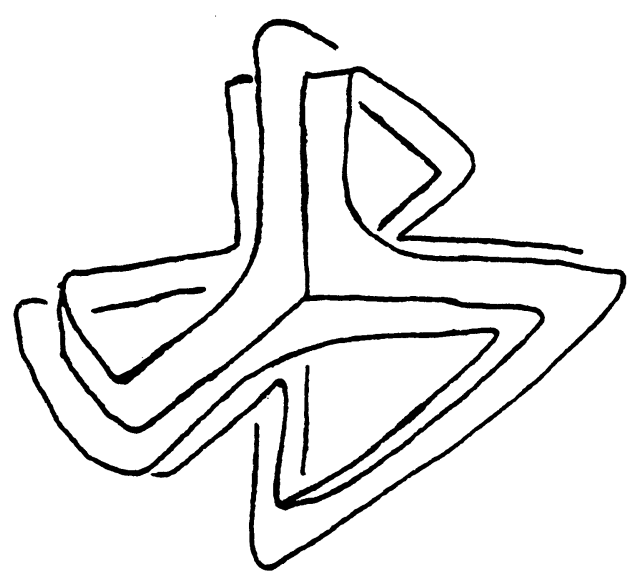

FIGURE 4 


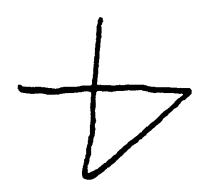

\section{FIGURE 5}

For $t \in[-1,1]$, there is a 2-disk in the plane $x_{j}=t$ transverse to the double-point curve so that the intersection of the immersed Möbius band with this disk "looks like" the union of the coordinate axes and an arc similar to $p_{j}$ as in Figure 5. (In the sequel we will say the coordinate arcs are joined in a $p_{j}$ fashion.) The phrase "looks like" is in quotations for when $x_{j}=0$, the intersection also consists of that part of the immersion contained in $\Gamma_{j}$. In fact, when $x_{j}=0$, Figure 5 is the intersection of the transverse disk $\Gamma_{j}(5)$ and the double-point manifold. The horizontal arc of Figure 5 when $x_{j}=1$ is the image of $\left\{t p_{j}{ }^{\perp}(0)\right\}_{t \in[-1,1]}$ in this disk. In order to close the boundary use the following:

For each $t \in[0,1]$ and each $j=1,2,3$, there is a 2-disk transverse to $p_{j}(t)$ so that the intersection of Boy's surface with this disk consists of a singular arc as seen in Figure 5. This intersection is called a standard slice. This statement may be thought of as an observation given by examining Figure 8, or as a method of parametrizing the remaining disk to be added. The latter notion requires explanation.

Figure 5 may be thought of as a frame in a movie. (There are actually three movies: one for each $j$.) A movie representation of three-space is a one-parameter family of 2-disks; the parameter is thought of as time. The movie in question has parameter given by the parametrization of $p_{j}(t)$; thus this movie depicts the immersion normal to the double point curve. The horizontal arc in any frame of this movie is some belt disk of the handle $h_{j, 0}$; the vertical is a belt disk of $h_{j, 1}$. For fixed $t$ these belt disks are spanned by $p_{j}{ }^{\perp}(t)$ and $e_{j+2}$ respectively. In Figure 6 , three stills from the $j$ th movie are shown. The stills have natural coordinatizations as shown.

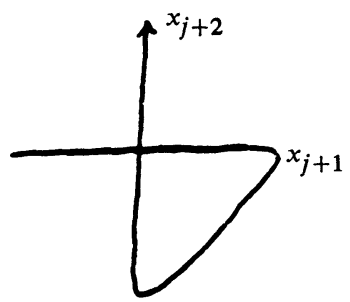

$p_{j}(0)=e_{j}$

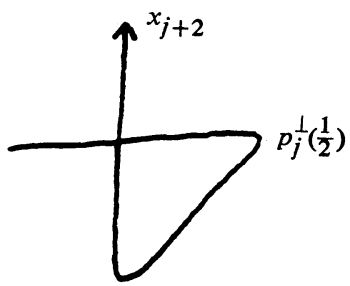

$p_{j}\left(\frac{1}{2}\right)$

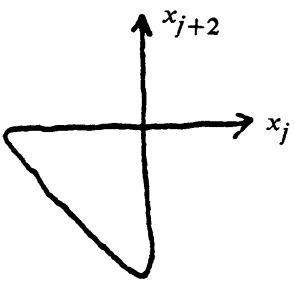

$p_{j}(1)=-e_{j+1}$ 


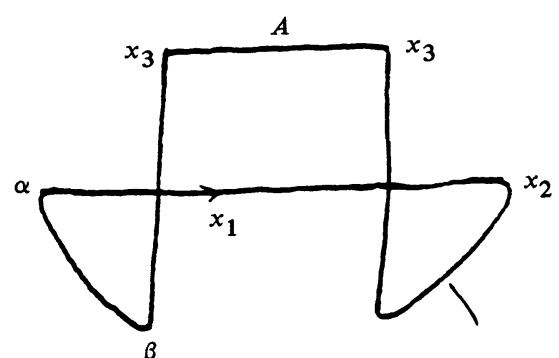

(a)

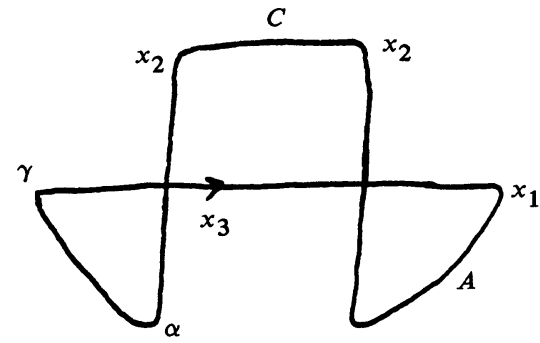

(c)

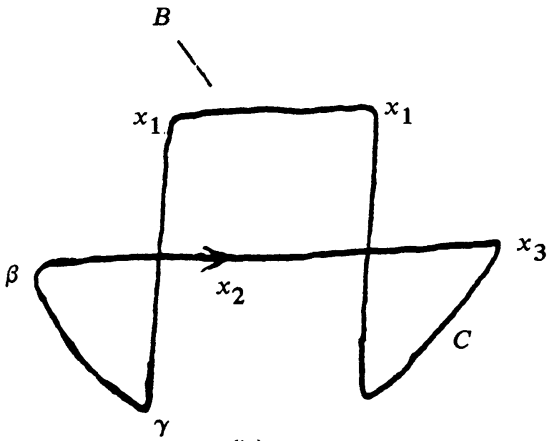

(b)

FIGURE 7

Two remarks are in order. First, the movies in Figure 6 do not give a complete parametrization of the remaining disk. Second, there are points of the immersion which appear in more than one movie. With regards to the second remark notice that the slice $x_{j}=1$ may be joined to the slice at $x_{j+1}=-1$ by joining the endpoints of their horizontal arcs. This is equivalent to slicing the immersion with a "bent knife" along any of the dotted arcs in Figure 3. Such slices appear in Figure 7.

The upper horizontal arc $A$ in Figure 7(a) is approximately the right diagonal arc in Figure 7(c). Other similar arcs are labeled with capital Roman letters. The points labeled in small Greek letters also coincide when the letters coincide. These facts are observed by examining the coordinates given. With regard to the first remark notice the points $\alpha=(-1,-1,0), \beta=(0,-1,-1)$, and $\gamma=(-1,0,-1)$ form the vertices of an equilateral triangle whose edges are shown as the left-hand diagonal arcs in Figure 7 of negative slope. Such a triangle has a face which is completely contained in the negative octant of 3-space. Thus it does not intersect the immersion except along its boundary. Figure 8 is a sketch of Boy's surface as viewed from the front and back.

In order to understand the immersion given in §III, one should try to imagine an intelligent 2-dimensional creature, who knows of the existence of Boy's surface trying to construct it. The construction above is given almost entirely via 2-dimensional cross sections. The most difficult step in this construction is adding the last triangle. 

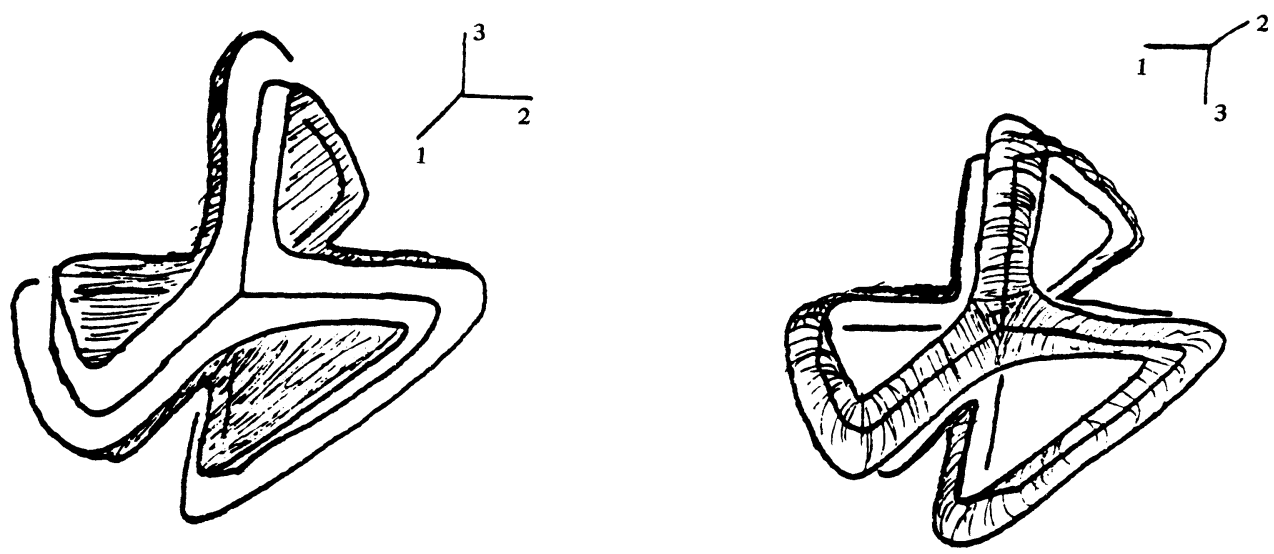

Figure 8

III. Construction of the generator of $\Pi_{3}^{s}$. A natural question arises when stable homotopy groups are identified with bordism groups of immersions: Does there exist an immersion with simple self-intersection sets representing a given homotopy class? In [5], we showed that classes on which the $(n+1)$-tuple invariant vanished could be represented without $n$-tuple points. Below we construct a generator of $\Pi_{3}^{s}$. By Eccles's theorem, this must have an odd number of quadruple points and the triple point data must be nontrivial. The generator given has exactly one quadruple point and relatively simple triple point data. In particular the triple point manifold is connected. All subscripts in this section are reduced modulo 4.

Proceed as above. For $j=1,2,3,4$, let

$$
\Gamma_{j}(r)=\left\{\left(x_{1}, x_{2}, x_{3}, x_{4}\right) \in \mathbf{R}^{4}: x_{j}=0,\left|x_{i}\right| \leqslant r\right\},
$$

and $\Gamma_{j}=\Gamma_{j}(1)$. The $\Gamma^{\prime}$ 's intersect pairwise in squares, any three intersect in a coordinate arc, and $\Gamma_{1} \cap \Gamma_{2} \cap \Gamma_{3} \cap \Gamma_{4}=(0,0,0,0)$. Let $p_{j}:[0,1] \rightarrow \mathbf{R}^{4}$ be the arc pictured in Figure 2, and let $p_{j}^{\perp}(t)$ be the indicated normal which lies in the plane spanned by $e_{j}$ and $e_{j+1}$. The normal space of $p_{j}(t)$ is spanned by $\left\{p_{j}^{\perp}(t), e_{j+2}, e_{j+3}\right\}$. Any two of these three vectors may be used to define an embedded 1-handle; parametrizations of these handles are easy to give (see the formulas for $h_{i, j}$ above). So to the four 0-handles, twelve 1-handles are attached; the cores of the 1-handles are the arcs, $p_{j}$, of triple points. In each of the 2-dimensional planes spanned by $\left\{e_{j}, e_{j+1}\right\}$ there is a disk bounded by a curve of double points; such a disk is roughly triangular shaped and is analogous to any one of the 2-handles seen in Figure 3. Let us call these disks black; each black disk serves as the core of two 2-handles.

At this stage four 0-handles, twelve 1-handles, and eight 2-handles have been attached. This construction yields an immersed solid torus $\left(D^{2} \times S^{1}\right)$ in 4-space. There is one quadruple point and the triple point set is an immersed circle. On the boundary of this torus there are six simple closed curves that are double points of the immersion. Since they are double points, pairs of these curves have the same image in $\mathbf{R}^{4}$. Figure 9(a) is a picture of the double point set of the immersion. 


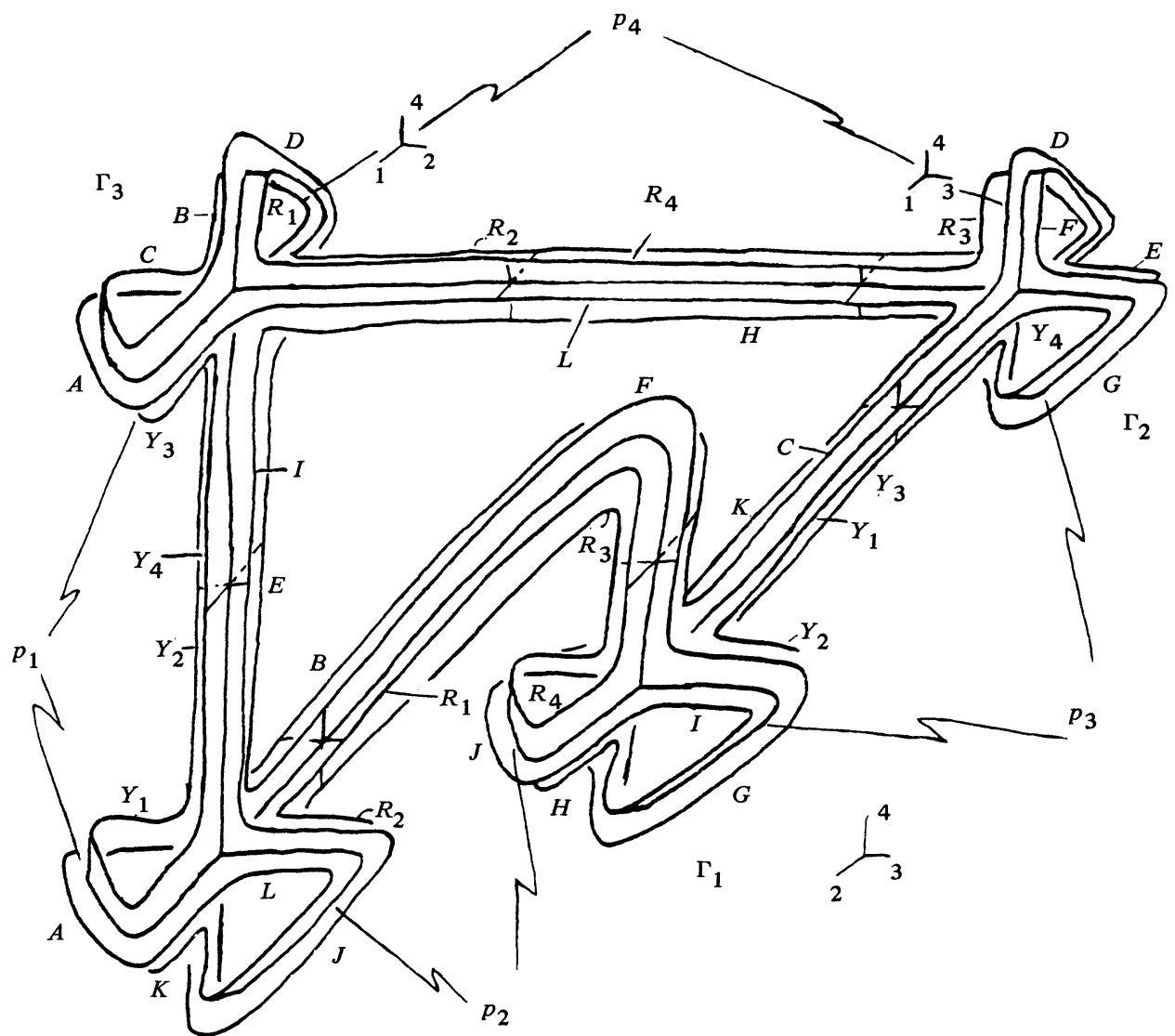

$\Gamma_{4}$

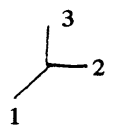

FIGURE 9(a)

The key to constructing immersed $n$-manifolds in $\mathbf{R}^{n+1}$ is to attach $k(n-k)$ handles whose cores intersect in a common $(n-k)$-disk embedded in $\mathbf{R}^{n+1}$. So the next stage of the construction is to add six 2-handles, two at a time; the attaching spheres of which are the double point curves discussed in the previous paragraph.

The images of these simple closed curves are parametrized in segments which run parallel to the $p_{j}$ 's. The "longest" of the curves is called the green curve. It is parametrized by the segments labeled $A$ through $L$. The segment $A$, for example, is the $\operatorname{arc} A(t)=p_{1}(t)+p_{1}^{\perp}(t)$. The point where segment $A$ meets segment $B$ will be denoted by $A B$, etc. The yellow curve is parametrized by $Y_{1}$ through $Y_{4}$, where $Y_{1}=p_{1}(t)+e_{3}$, and so forth. This is the curve which appears on the far left and far right of Figure 9(a). The red arc is parametrized by segments $R_{1}$ through $R_{4}$; for example $R_{1}=p_{4}(t)+e_{2}$. This arc is at the top and bottom of Figure $9(\mathrm{a})$. The 


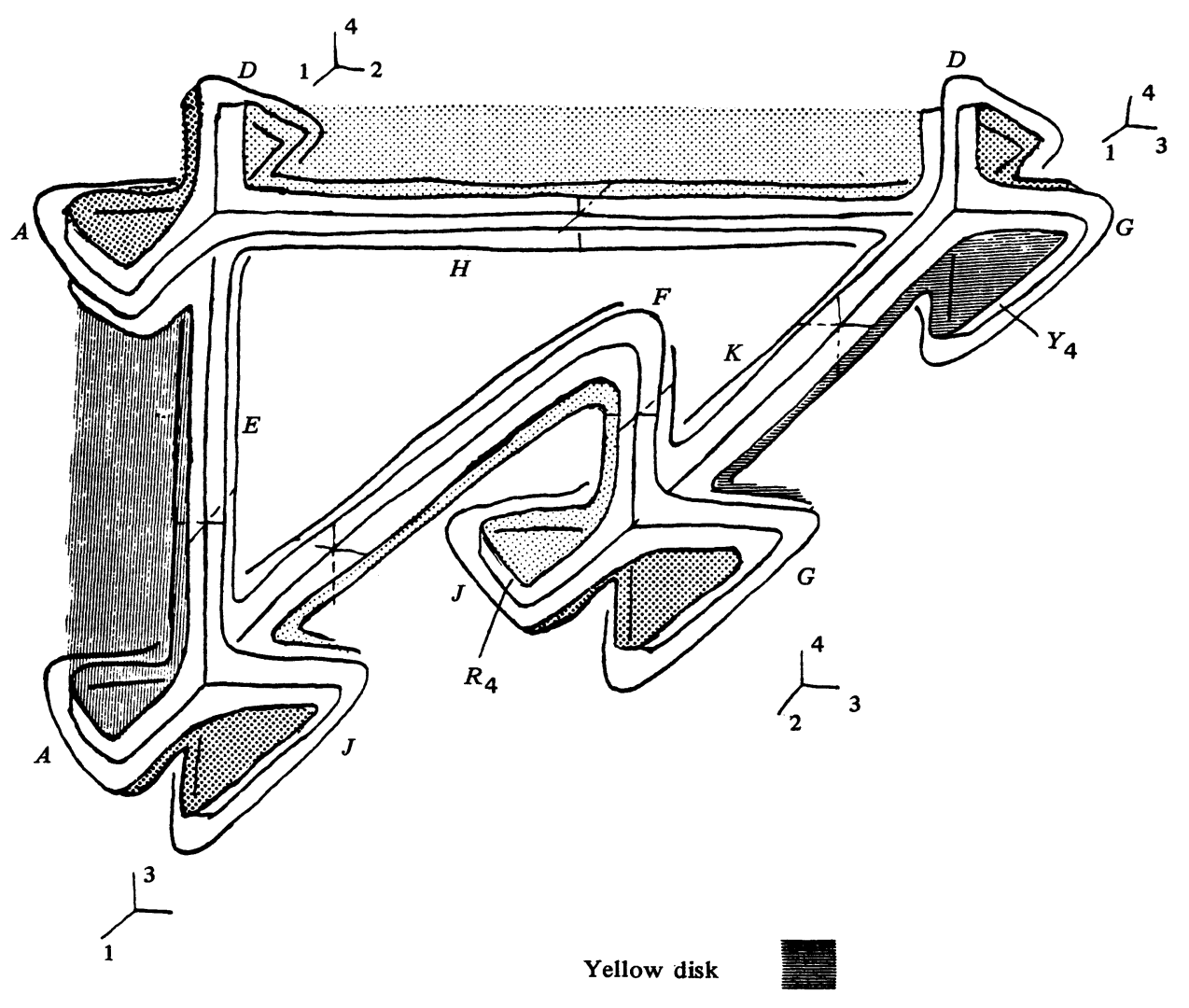

Red disk

Green disk
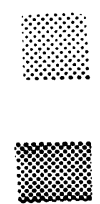

FIGURE 9(b)

chromatic nomenclature here is used to suggest Figures 9(a), (b), and (c) should appear in color. It is much easier to visualize with such contrast added. (See Figure 9(b).)

The cores of the 2-handles added have images the green disk, the yellow disk, and the red disk. Of these the green disk is hardest to visualize; the yellow and red are strictly analogous. Let us attach the "easy" 2-handles first. The image of the yellow disk in $\mathbf{R}^{4}$ has a shape as in Figure 10(a). The two "lobes" or delta shaped regions are "bounded" by $Y_{2}$ and $Y_{4}$; these boundaries are parallel to $p_{3}$. The long strip has edges running parallel to $p_{1}$. This disk also appears on the right of Figure 9(b). On the left of Figure 9(b), the disk is distorted somewhat more than it is on the right. The red disk has a similar shape with the lobes being bounded by $R_{2}$ and $R_{4}$. The least distorted view of this disk appears at the bottom of Figure 9(b). 


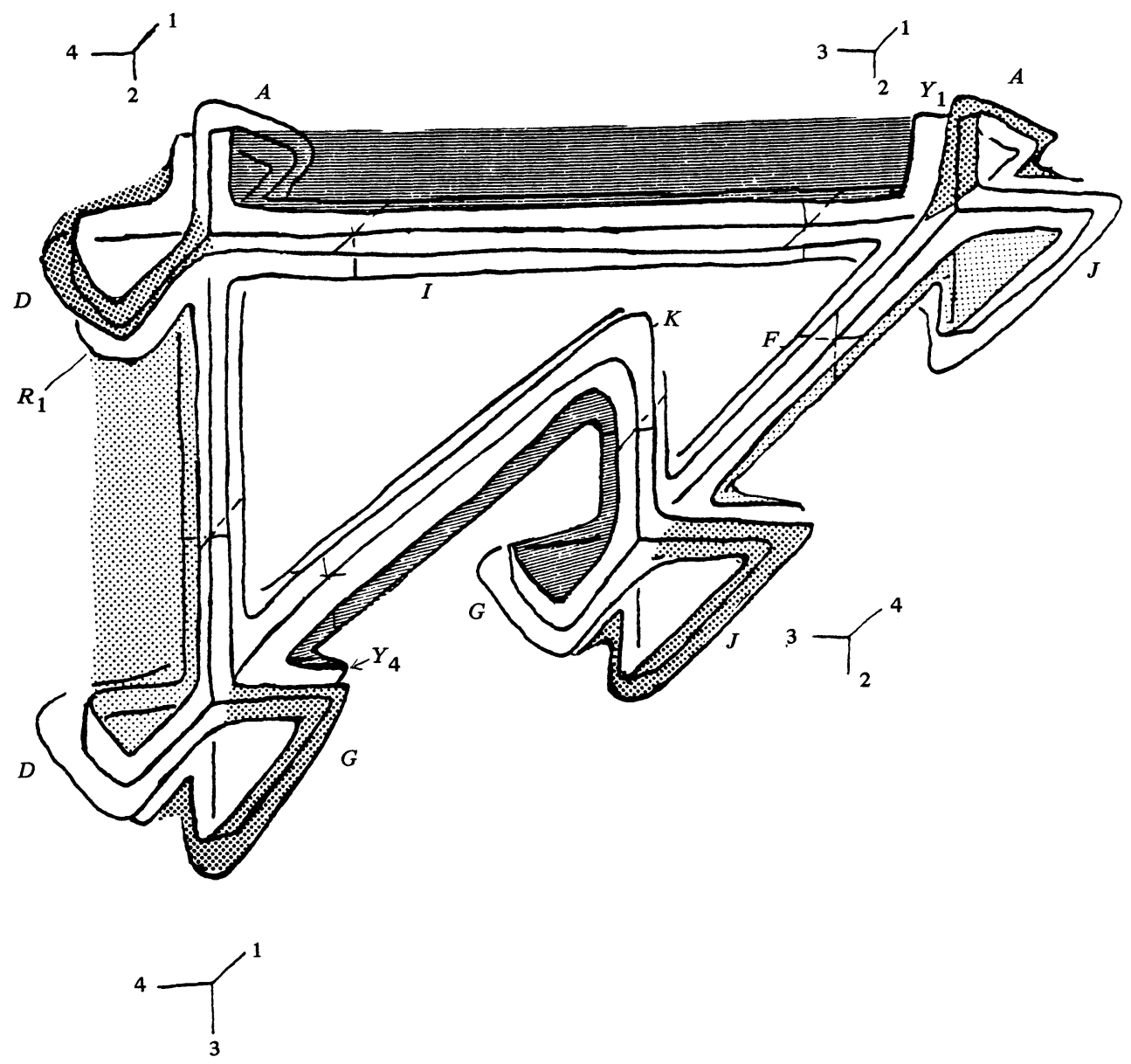

FiguRE 9(c)

The unfortunate feature of the green disk is that it cannot be seen all at once. The $\operatorname{arcs} C, F, I$, and $L$ "bound" lobe-shaped regions of the disk. From the open end of the $C$-lobe a thin rectangular strip with edges $B$ and $D$ extends (the $(B, D)$ tail of the $C$-lobe). Similarly from the $F$-lobe a strip with "horizontal" edges $G$ and $E$ extends, from the $I$-lobe a strip with edges $H$ and $J$ extends, and from the $L$-lobe a strip with edges $A$ and $K$ extends. The "back" edges of these four strips have the vertices $A B, D E, G H$, and $J K$ in common. The four edges $(A B, D E),(D E, G H)$, $(G H, J K)$, and $(J K, A B)$ form the edges of a square

$$
s:[0,1] \times[0,1] \rightarrow R^{4}, \quad s(u, v)=(-u,-v,-(1-u),-(1-v)) .
$$

The four lobes, four strips, and square glue together to form the green disk; the shape of which is seen in Figure 10(b). In Figure 9(b) most of this disk is shown; Figure $9(\mathrm{c})$ gives a back view so that the edges of the green square, $s([0,1] \times[0,1])$, are seen easily. 


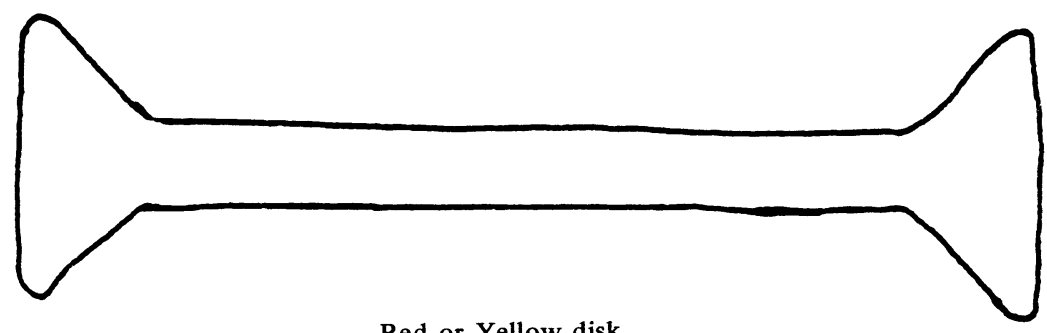

Red or Yellow disk

(a)

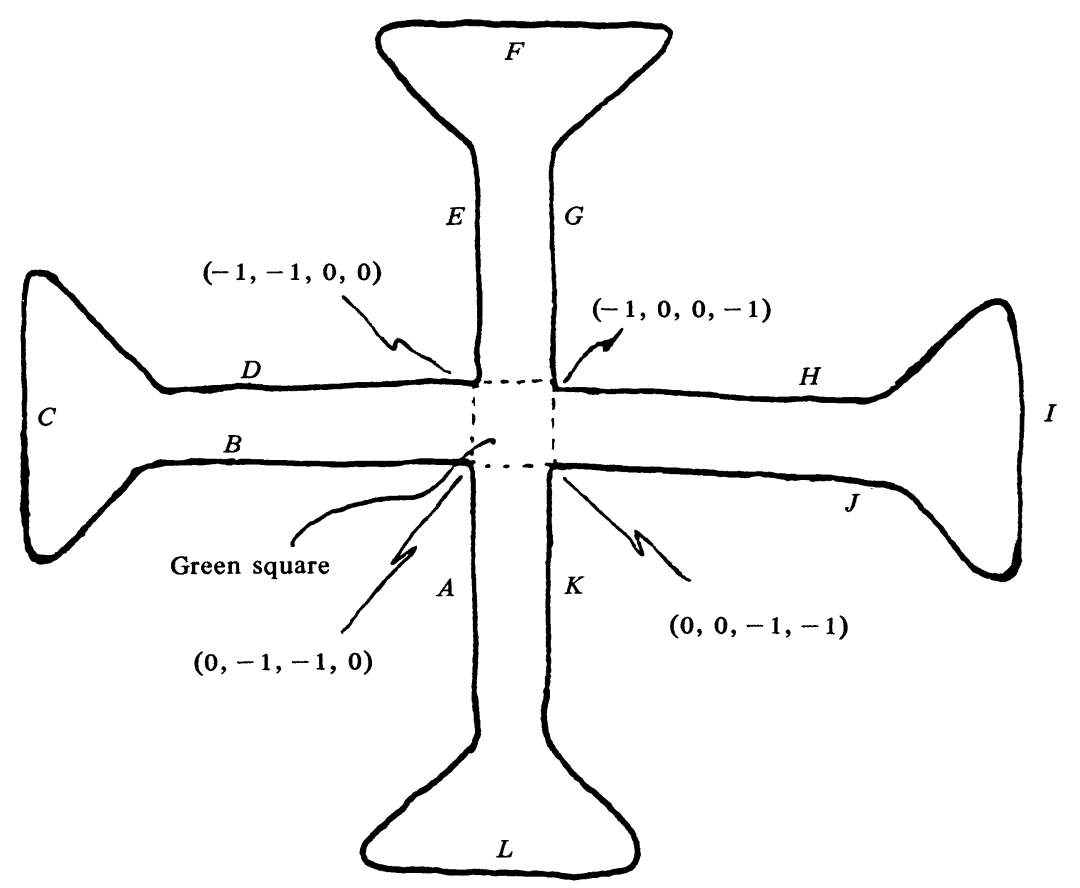

Green disk

(b)

Figure 10

Recall, each of the green, yellow, and red disks serves as the core of two 2-handles. These handles determine a surgery on the solid torus, so the boundary of the new manifold is a collection of 2-spheres. Now is an opportune time to count the number of 3-handles to be added. There are four 0-handles, twelve 1-handles, and fourteen 2-handles so far. Thus since the Euler characteristic of a closed 3-manifold is zero, there must be six 2-sphere boundary components. 
Before adding the remaining six 3-handles some remarks are in order. The double point surface is now closed and is easily computed to be $\mathbf{R} \mathbf{P}^{2}$. The preimage of this surface in the 3-manifold is an immersed 2-sphere. The triple-point manifold is a closed curve. In the theorem below it is shown that the triple-point set and the quadruple-point set necessitate that the completed immersion represents a generator of $\Pi_{3}^{s}$.

Four of the six 3-handles to be added are easily seen. They are added "below" the four red and yellow 2-handles as is indicated in Figure 9(b). (We use prepositions such as "below" and "over" with some caution.) The remaining two 3-handles will be referred to as the red and yellow balls due to the role that the red and yellow disks play in their construction. This terminology should be contrasted: thus far we have named the green curve (parametrized by $A-L$ ), the green disk (bounded by said curve), the green square $s([0,1] \times[0,1])$, the red and yellow curves and disks, and the red and yellow balls.

The construction of the red and yellow balls is motivated by three notions. First, for $j=1$ or 4 and $t \in(0,1)$, there is a 3-ball in the hyperplane $x_{j}=t$, normal to the triple-point curve so that the double point manifold intersects this 3-ball in a graph that is similar to the image of the triple-point set in $\Gamma_{j}(R)(R>1$ some number). Second, in a disk normal to any double-point the immersed manifold with boundary appears as a pair of coordinate arcs; the intersection point is the given double point. Third, the boundary of the red and yellow 3-balls are almost completely seen by looking at Figures 9(b) and 9(c); the missing pieces of the boundaries appear "over" the green square, $s(u, v)=(-u,-v,(u-1),(v-1))$ for $u, v \in[0,1]$.

The red and yellow balls are constructed simultaneously in pieces. To construct the first pieces, slice the immersion with four "bent knives". For $j=1,2,3$, and 4, the $j$ th slice contains a hyperplane normal to $p_{j}(\varepsilon)=(1+\varepsilon) e_{j}$ and a hyperplane normal to $p_{j}(1-\varepsilon)=-(1+\varepsilon) e_{j+1}$ where $\varepsilon>0$ is small. These slices are analogous to the slices (Figure 7) made on Boy's surface along the dotted arcs of Figure 3. Figure 11 indicates the structure of the double-point manifold in these slices; coordinates are given at the triple-points. The points labeled $Y_{1} Y_{2}, A B, L A$, etc. indicate that these points are approximately the endpoints of the segments of the appropriate colored arcs. Thus any two of these cross sections contain common arcs of double points.

Since the structure of the double-point manifold at $x_{j}=1+\varepsilon$ is similar to the structure of the triple-point manifold at $x_{j}=0(j=1$ or 4$)$, the nonsingular set in the slice $x_{j}=1+\varepsilon$ resembles the double-point set in the slice $x_{j}=0$. This is the notion of standard slices engendered since at $x_{j}=1$ the nonsingular set has standard form. Standard slices do not work for $j=2$ or 3 . Standard slices together with other data yield Figures 12(a) and 12(d).

Figures 12(a) and 12(d) indicate that embeddings of certain pieces of the red and yellow balls are given by a movie representation (see §II, Figure 6) of the normal space of $p_{j}$ when $j=1$ or 4 . That is, the slice at $x_{j}=1+\varepsilon$ is rotated to the slice at $x_{j+1}=-1-\varepsilon$, and a 1-parameter family of surfaces is engendered in this fashion. A 
coloring of the surfaces at $x_{j}=1+\varepsilon$ yields a coloring of the surfaces in any of the balls normal to $p_{j}(t)$; such a ball is spanned by $e_{j+2}, e_{j+3}, p_{j}^{\perp}(t)$.

When $j=2$ or 3 , the double-point surface at $x_{j}=1+\varepsilon$ does not look the same as the triple-point curves at $x_{j}=0$. However, since Figures 11(a) and 11(b) have the green arc $(L A, K L)$ in common, (and similarly Figures 11(d) and 11(c) share the green arc $(E F, F G))$, the structure of the nonsingular sets in the (b) and (c) figures may be extrapolated to yield Figures 12(b) and (c). These extrapolations in turn yield more data to complete the figures 12(a) and 12(d). (These data were alluded to two paragraphs above.) Furthermore, the symmetry of the (b) and (c) figures of 12 reflects the movie representation of these pieces of the red and yellow balls.

The preceding few paragraphs suggest that Figures 12(a)-(d) are the end product of an evolutionary process. That is, make certain extrapolations to get portions of the (a) and (d) figures, and use these to interpolate the structure of the (b) and (c) figures. Then go back to the (a) and (d) figures and try to complete them. Finally, interpret these figures as the initial and final frames of movies. This being done alternate descriptions of the red and yellow balls are given.

Let $\alpha$ be a point on the green disk not contained in the green square. Thus $\alpha$ is any green point of Figure $9(\mathrm{~b})$ or (c). Let $D(\alpha)$ be a disk normal to the green disk at $\alpha$ of small radius. Since $x$ is not in the green square, $D(\alpha)$ is spanned by $e_{j}$ for some $j$ and some other vector. For example if $\alpha$ is on the $C$-lobe, then $D(\alpha)$ is spanned by $e_{3}$ and $e_{4}$. If $\alpha$ is in the $B D$-tail of the $C$-lobe then $D(\alpha)$ is spanned by $e_{3}$ together with a vector $\alpha^{\perp}$ which is written in terms of $e_{1}, e_{2}$, and $e_{4}$. These two normal vectors yield an isomorphism $\omega$ from $D(\alpha)$ to the standard unit disk in $\mathbf{R}^{2}$. The normals go to unit vectors under $\omega$, and the coordinate arcs are the images of belt disks of the "green cored" 2-handles. (Recall, the green disk is the image of the cores of two 2-handles.) If $\alpha \in \Gamma_{j}$, then $\omega\left(e_{j}\right)$ is the unit $x$-vector if and only if $j$ is odd. Color the endpoints of the coordinate arcs either red or yellow as follows: The points $(1,0)$ and $(0,-1)$ are yellow while $(0,1)$ and $(-1,0)$ are red. Notice that this color scheme agrees with the color scheme of Figure 12.

Now suppose $\alpha \in \Gamma_{j}$ for $j=1$ or 3 . Then according to Figure 12 the positive $y$-axis is joined to the negative $x$-axis in a $p_{j}$-fashion. This joining occurs in a disk of larger radius than $D(\alpha)$. When the radius of the normal disk is increased, the negative $y$-axis remains in the hyperplane $\Gamma_{j}$. In fact, by Figure 9 (c) one would expect that the yellow ball goes "below" the lobe and tail of the green disk within $\Gamma_{j}$. If $j$ is even, rewrite this paragraph interchanging the words $x$-axis and $y$-axis and the words red and yellow. In this fashion a large portion of the red and yellow balls are added. This notion agrees with the notion used to draw Figure 12.

Before gluing the pieces of the red and yellow balls together over the green square, turn now to coloring the boundary of the ambient manifold. Work with Figures 9(b) and 9(c) keeping matters consistent with the preceding remarks and with Figures 12(a)-(d). Pretend that both sheets of the green disk may be seen in Figures 9(b) and 9(c). In this way any of the green curves on the boundary of the solid torus represents a genuine barrier to passing from one region to the next. Also pretend that surfaces drawn have thickness. The red sphere then consists of the set of all 
GENERALIZING BOY'S SURFACE
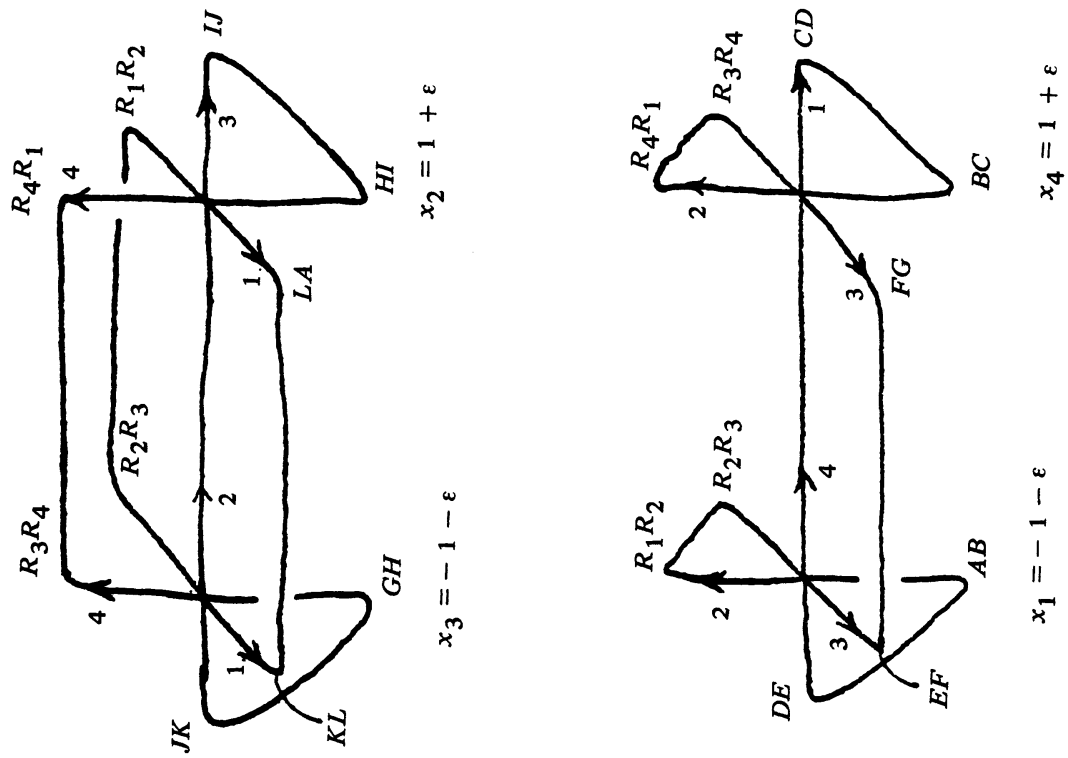

$\hat{e}$

()
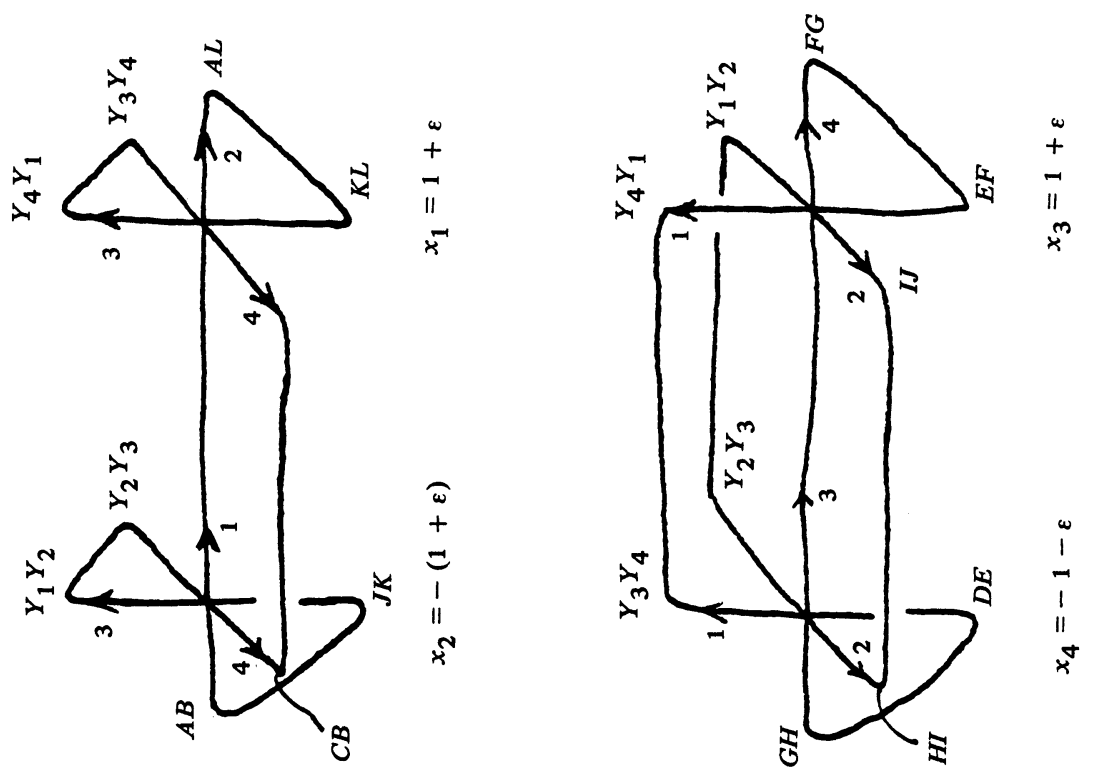
Yellow Ball

Red Ball
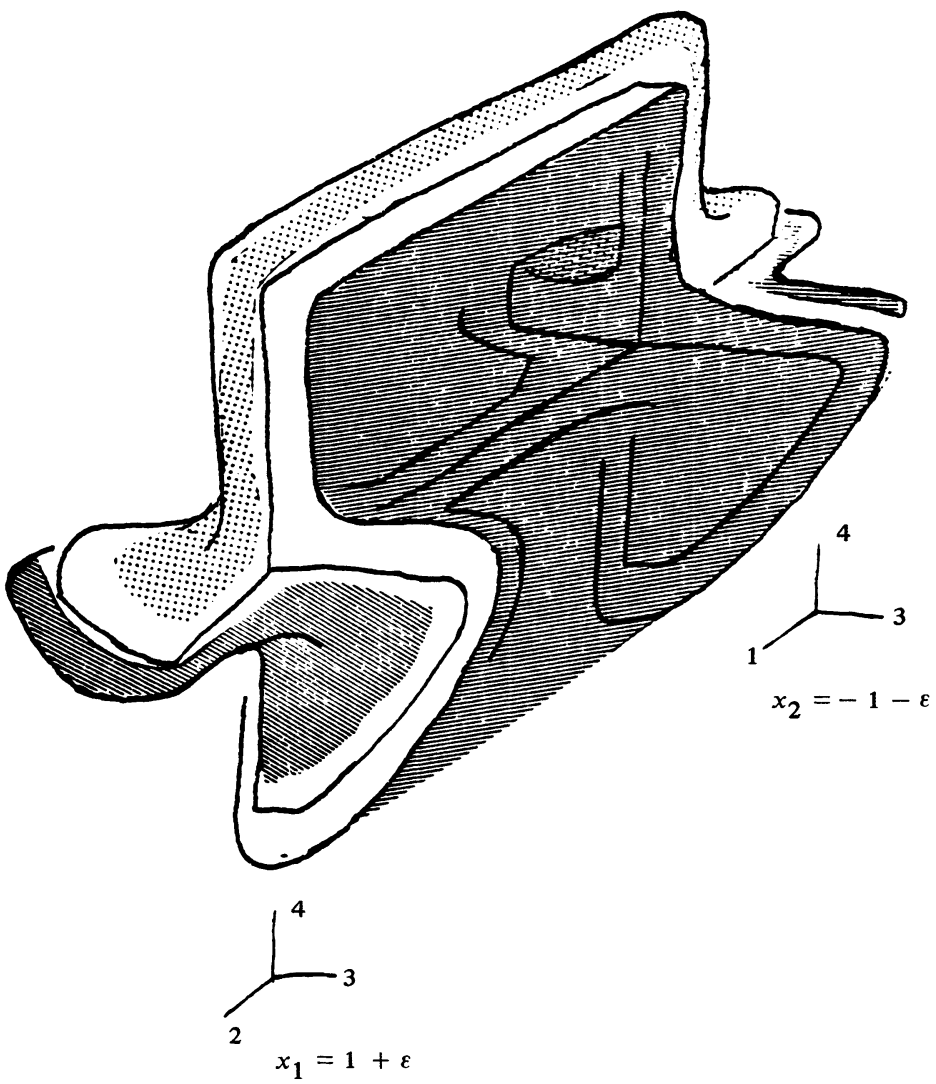

Figure 12(a)
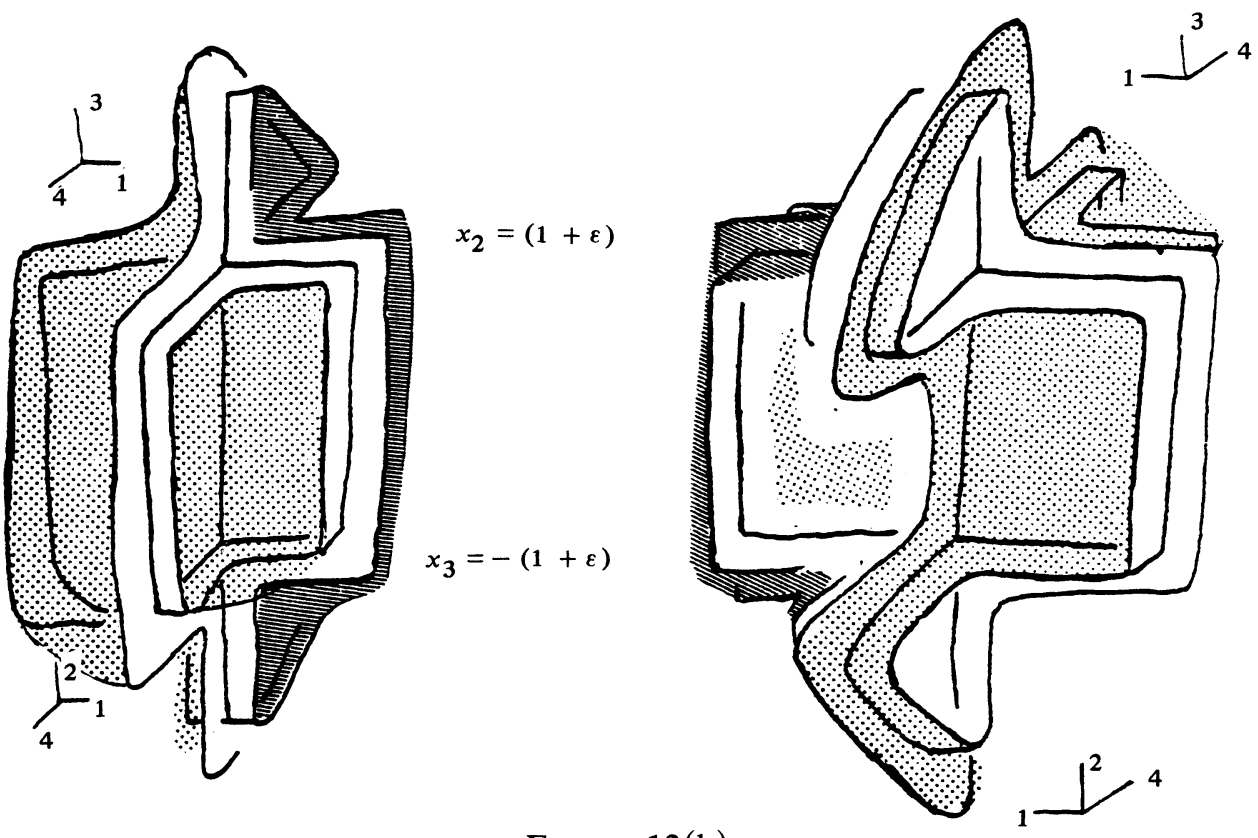

Figure 12(b) 
GENERALIZING BOY'S SURFACE
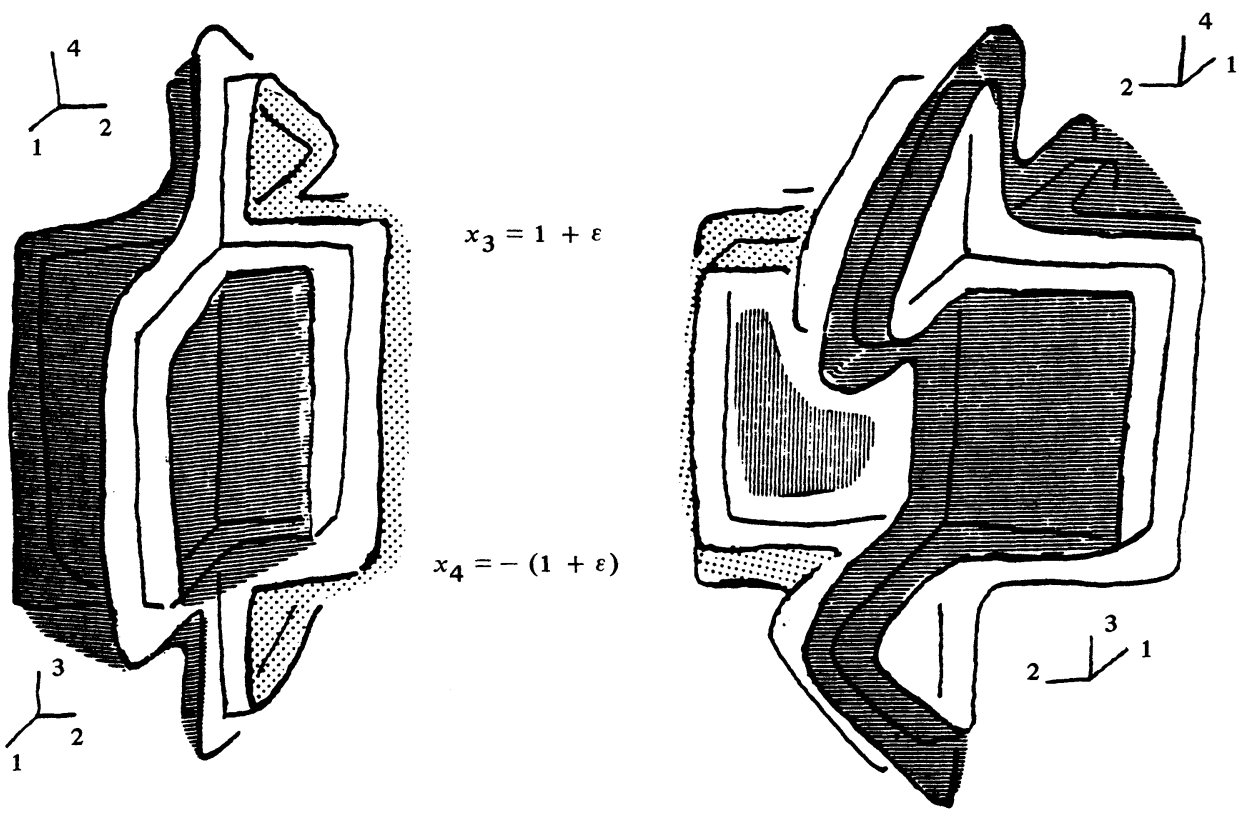

FigURE 12(c)

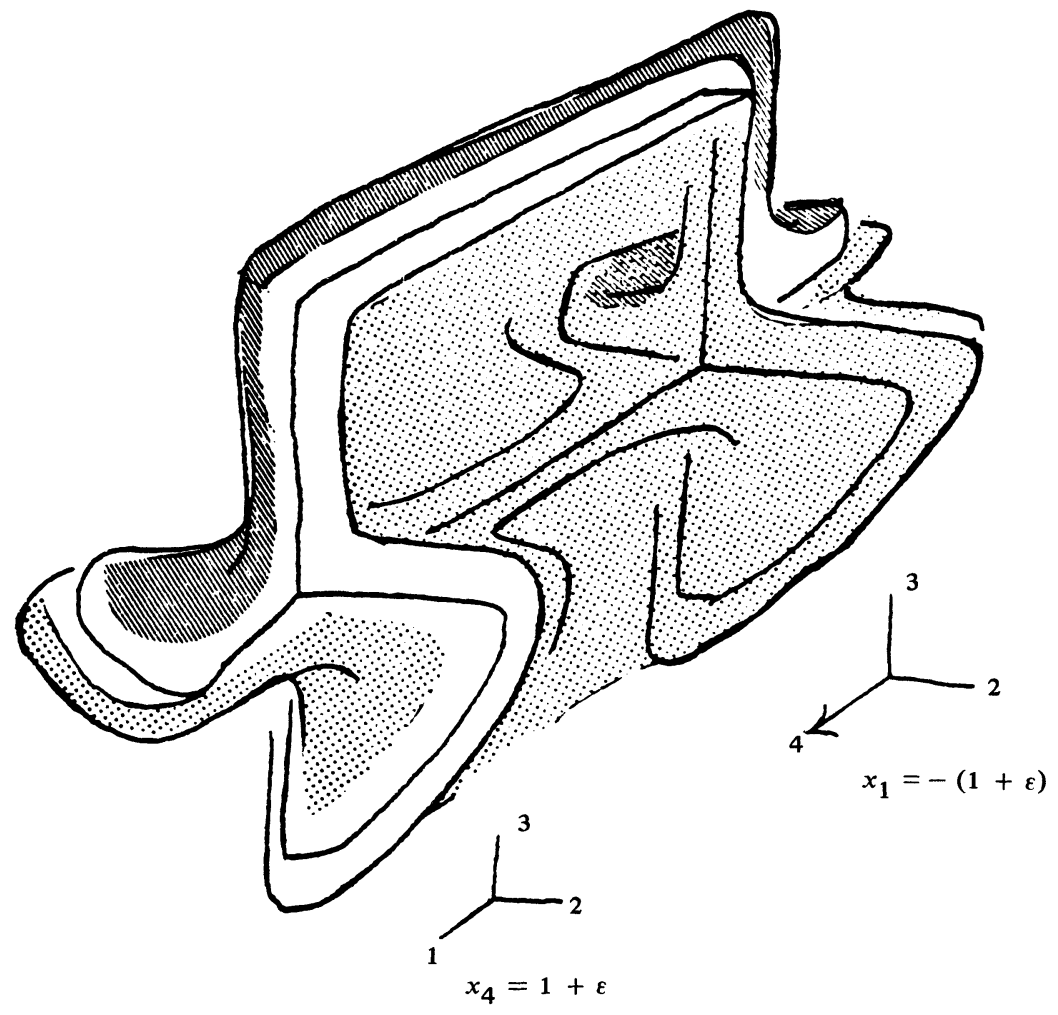

FIGURE 12(d) 


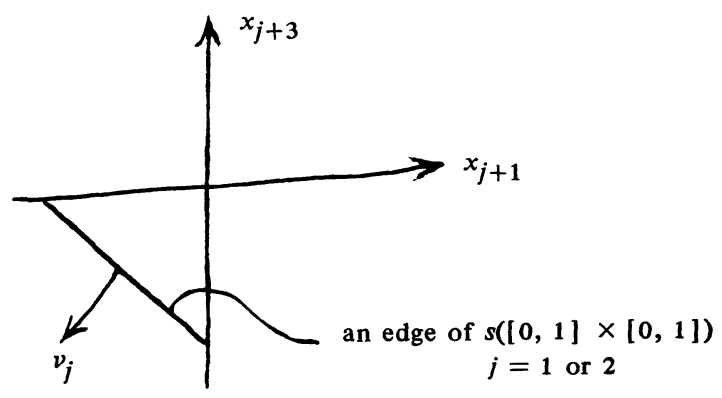

FIGURE 13

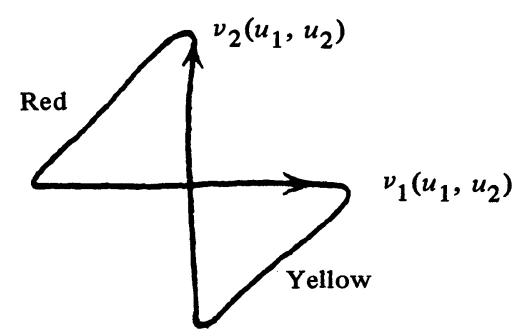

Figure 14

points in Figure 9 which may be joined to a point on the red disk by an arc which does not cross any green curve. A similar definition yields the yellow sphere. Of course, these are not yet spheres as the green square has been neglected.

So what happens over the green square? Each of the four edges of $s([0,1] \times[0,1])$ are contained in some hyperplane, and according to the preceding discussion there are two vectors normal to any edge which span the belt disks of the 2-handles. One such vector is $e_{j}$ if the edge is contained in $\Gamma_{j}$, and the other is $-\left(e_{j+1}+e_{j+3}\right)$ (see Figure 13). So the given normal vectors do not agree from edge to edge. For $\left(u_{1}, u_{2}\right) \in(\varepsilon, 1-\varepsilon) \times(\varepsilon, 1-\varepsilon)$ define

$$
\nu_{1}\left(u_{1}, u_{2}\right)=-\frac{1}{\sqrt{2}}\left(e_{1}+e_{3}\right) \text { and } \nu_{2}\left(u_{1}, u_{2}\right)=-\frac{1}{\sqrt{2}}\left(e_{2}+e_{4}\right) \text {. }
$$

In the plane spanned by $\nu_{1}$ and $\nu_{2}$ define $+\nu_{1}$ and $-\nu_{2}$ to be yellow and $-\nu_{1}$ and $+\nu_{2}$ to be red. Now join the red points in the second quadrant, and join the yellow points in the fourth quadrant. So for each $u_{1}, u_{2} \in(\varepsilon, 1-\varepsilon)$ the normal plane contains a Figure 8 as in Figure 14. The product of this (red) yellow arc and the green square is called the (red) yellow box. These boxes remain to be attached to the pieces of the red and yellow balls already in place.

The red and yellow boxes are embeddings of $[0,1] \times[0,1] \times[0,1]$ which are affine except near the faces where we have taken the trouble to round some corners. Think 
of the top and bottom of each box as being tinted green; these are the faces "parallel" to the green square. In each of Figures 12(a)-(d), one sees one full side of a red or yellow box, and two edges of a box of the opposite color; so not all of the red and yellow sides appear. Those sides of the same color which do appear are parallel. The remaining sides simply dip out of the given hyperplane. This completes the construction.

THEOREM. The construction above yields an immersion of $S^{3}$ in $\mathbf{R}^{4}$ which represents a generator of $\Pi_{3}^{s}$.

Proof. That the manifold is the 3-sphere follows since either pre-image of the green circle (parametrized by $A-L$ ) is a $(1,1)$-torus knot on the boundary of the solid torus. Since the immersion has one quadruple point, it represents a class that is odd in $\Pi_{3}^{s} \cong \mathbf{Z} / 24$. According to Eccles's theorem (the first theorem quoted), the triple point set detects the 3-torsion. But the triple-point matrix is the lower right block of the product of the matrices

$$
\left(-e_{4}, e_{2}, e_{3}, e_{1}\right)\left(e_{1}, e_{2}, e_{4},-e_{3}\right)\left(e_{1}, e_{3},-e_{2}, e_{4}\right)\left(e_{2},-e_{1}, e_{3}, e_{4}\right) .
$$

Under a suitable choice of the normal basis this is an unsigned cycle of order 3 . Thus the triple-point invariant,

$$
\psi_{3}: \Pi_{3}^{s} \rightarrow \Pi_{4}^{s}\left(D_{3} S^{1}\right) \cong \mathbf{Z} / 2 \oplus \mathbf{Z} / 3, \quad(\text { see }[14])
$$

when evaluated on the given immersion is nontrivial modulo 3 . Therefore this immersion generates. This completes the proof.

QUESTIONS. 1. Is the immersion constructed regularly homotopic to a "capped-off" 2-sphere eversion? Try turning the boundary of the red cube inside out.

2. The yellow and red arcs may be closed in a fashion which yields four symmetric cross sections. Doing so requires a yellow square and a red square. Does either process yield a closed immersion with 4-fold symmetry?

\section{BIBLIOGRAPHY}

1. T. Banchoff, Triple points and surgery of immersed surfaces, Proc. Amer. Math. Soc. 46 (1974), 407-413

2. W. Boy, Über die curvature integra und die topologie geschlossener flaschen, Math. Ann. 57 (1903), $151-184$.

3. J. S. Carter, Surgery on immersions: a geometric approach to stable homotopy, Dissertation, Yale University, 1982.

4. Surgery theory of immersions, Proc. Evanston Conf. on Homotopy Theory, Haynes Miller and Stewart Priddy (eds.), Contemporary Math., vol. 19, Amer. Math. Soc., Providence, R. I., 1983, pp. 23-37.

5. Surgery on codimension one immersions in $\mathbf{R}^{n+1}$ : removing $n$-tuple points, Trans. Amer. Math. Soc. 298 (1986), 83-101.

6. P. J. Eccles, Multiple points of codimension one immersions, Ulrich Koschorke (ed.), Top. Sympos. Siegen, Lecture Notes in Math., vol. 788, Springer-Verlag, Berlin and New York, 1979, pp. 23-38.

7. Multiple points of codimension one immersions of oriented manifolds, Math. Proc. Cambridge Philos. Soc. 87 (1980), 213-220.

8. Codimension one immersions and the Kervaire invariant one problem, Math. Proc. Cambridge Philos. Soc. 90 (1981), 483-493.

9. M. H. Freedman, Quadruple points of 3-manifolds in $S^{4}$, Comment. Math. Helv. 53 (1978), 385-394. 
10. D. Hilbert and S. Cohn-Vossen (Translated by P. Neményi), Geometry and the imagination, Chelsea, New York, 1952, pp. 320-321.

11. J. F. Hughes, Invariants of regular homotopy and bordism of low-dimensional immersions, Dissertation, Univ. of California, Berkeley, 1982.

12. D. S. Kahn and S. B. Priddy, On the transfer in the homology of symmetric groups, Math. Proc. Cambridge Philos. Soc. 83 (1978), 91-101.

13. The transfer and stable homotopy theory, Math. Proc. Cambridge Philos. Soc. 83 (1978), $103-111$.

14. Ulrich Koschorke, Multiple points of immersions and the Kahn-Priddy Theorem, Math. Z. 169 (1979), 223-236.

15. ___ Vector fields and other vector-bundle morphisms - A singularity approach, Lecture Notes in Math., vol. 847, Springer-Verlag, Berlin and New York, 1981.

16. Ulrich Koschorke and Brian Sanderson, Self intersections and higher Hopf invariants, Topology 17 (1978), 283-290.

17. J. Lannes, Sur les immersions de Boy, Preprint, 1982.

18. N. Max and T. Banchoff, Every sphere eversion has a quadruple point, Contributions to Analysis and Geometry, D. N. Clark, G. Pecelli, and R. Sacksteder (eds.), John Hopkins Press, Baltimore, Md., 1981.

19. P. Vogel, Cobordisme d'immersions, Ann. Sci. École Norm. Sup. (4) 7 (1974), 317-358.

20. R. Wells, Cobordism groups of immersions, Topology 5 (1966), 281-294.

Department of Mathematics, University of Texas at Austin, Austin, Texas 78712

Current address: Department of Mathematics, Lake Forest College, Lake Forest, Illinois 60045 